\title{
TOOLS FOR BIOMEDICAL DATA ARCHIVING IN UKRAINIAN GRID INFRASTRUCTURE
}

\author{
Oleksandr Sudakov, Andrii Salnikov, levgen Sliusar, Oleksandr Boretskyi \\ Information and Computer Centre, National Taras Shevchenko University of Kyiv, \\ Ukraine, Kyiv, 4D Glushkova prosp., e-mail: cluster@cluster.kiev.ua, http://grid.org.ua
}

\begin{abstract}
Tools for archiving and extraction of data in Ukrainian National Grid for end-users' applications are proposed, implemented and deployed for practical applications in medical imaging, non-linear dynamics, and molecular biology. Proposed tools provide the facilities to utilize large distributed storage space in grid infrastructures for different practical tasks including desktop applications. Tools may be successfully used even when on client platforms it is impossible to setup grid middleware, use web browser interfaces or grid security infrastructure authentication. Tools consist of extensible client compatible with different software and hardware platforms; web service for data transfer; web service for transparent data replication on grid storage elements. Copyright $\mathbb{C}$ Research Institute for Intelligent Computer Systems, 2013. All rights reserved.
\end{abstract}

Keywords: Grid, Replication, Web service, Client, Storage, Protocol, Graphics, Anonymization, Image, DICOM.

\section{INTRODUCTION}

Computing Grids [1] are geographically distributed means for high-throughput computing and large data storage in scientific, industrial, commercial and others branches of activity. Ukrainian National Grid (UNG) infrastructure [2] was created in 2006 and is being used in number of applied projects like molecular dynamics simulations [3], neuroscience [4], non-linear dynamics [5] etc. In all these applications large amount of data is generated by imaging devices, simulation or data processing software. Grid infrastructure provides consolidated capabilities for data storage, high performance computing, data replication, user interfaces and other services. Grid also provides means for data security, reliability and high throughput access. The idea to transparently use large distributed grid storage space to store data from end-user server and desktop applications is very attractive because it eliminates the necessity to create big data centers at the user side, reduces costs for administration etc. Such transparent grid storage system has features of cloud or big-data storages and should be implemented using grid services.

The most common way to access grid resources is setup of grid middleware on client platform, via web interfaces or accessing third party grid access platforms. Unfortunately in many cases transparent usage of grid infrastructure from desktops, servers and devices is not easily possible. For example, usually it is not possible to setup grid middleware on the medical imaging device hardware, web interface is no usually good to transfer large amount of data, it is not always possible to provide personal certificates for all possible users etc. Many tasks may have other special requirements.

In present work we introduce a set of tools for working with data in grid infrastructure using conventional web services and extensible clients: RAPTOR replication and data exchange service, web-interface for AMGA metadata catalogue and grid-enabled DICOM client. These tools may be easily used for application of grid infrastructure to work with medical and other data on workstations without grid middleware on large number of hardware and software platforms. Proposed tools proved to be rather universal and efficient for wide number of application including medicine, dynamical simulations and molecular biology.

\section{UNG GRID DATA STORAGE REQUIREMENTS}

The most specific requirements for grid storages in UNG arise from medical applications [6]. For a last few years several projects concerned with medical applications of UNG have arisen. These projects are held in the virtual organizations (VO) [7] medgrid [8], telemed and others and are devoted to sampling, archiving, reconstruction, recognition, fusion etc. of electrocardiography 
(ECG), electroencephalography (EEG), nuclear medicine, ultrasound (USI) and other modalities data. Grid infrastructure provides consolidated capabilities for data storage, high performance computing, data replication, user interfaces and other services.

Data-intensive and computation-intensive medical applications have some special features. 1) Medical data is usually being transferred in special formats, like DICOM (Digital and Image Communication in Medicine) [9]. 2) Medical images contain personal patients' information and transfer of these data without patients' consent is legally prohibited. So it should be impossible to recover patients' personal data from images stored in the grid infrastructure for persons who are not authorized. 3) Medical images series may have large size up to about 1 GByte per examination and its transfer may require relatively large time and evidently may be aborted. 4) Medical images usually have large pixel depth (12-16 bits), may contain additional data and thus require special visualization techniques not available in conventional web browsers. 5) Jobs processing in grid usually introduce high latencies so client software should take this into account. 6) Credential management procedures for grid environment like personal grid certificates enrollment and renewal are relatively complicated and not usually acceptable for patients and physician thus additional simplified authentication methods should be used.

There are implementations of grid tools for working with DICOM images, like MDM (Medical Data Management) [10]. All these tools assume that grid middleware is installed on the client machine or there is DICOM PACS server available for physician. Unfortunately most Ukrainian medical centers still lack PACS servers and DICOM images are created on physician workstation form other formats. It is impossible to install and configure gLite middleware on client MS Windows-based workstations and there are many other reasons why MDM or other grid DICOM tools is hardly to be used now.

Other UNG grid applications have not so strict requirements and tools designed for medical data may be easily adapted to them.

\section{MEDICAL DATA STORAGES IN UNG}

Taking into account all the requirements stated above the following medical data storage approach was suggested for UNG. All data is physically stored on the replicated storage elements (SE). Replica locations are managed by highly available LFC (LCG File Catalog) grid services; data access to SEs is controlled by SRM (Storage Resource
Manager) [11] grid services; clients accesses are performed by specially designed web services that interact with SRM. SRM standard provides implementation-independent interface to storage management functions e.g. third-party data transfers, storage space tokens and reservations, nearline storage interaction and access control. All data on SEs is stored in anonymized form. It means that all patients' personal information should be wiped from the metadata on clients before it gets transferred to grid. All data on grid storages may be accessed by authorized VOs members and may be used for processing on grid computing elements (CE).

A decision was made that all the Storage Elements which are devoted to medical data in the UNG should support SRM control protocol. Currently, all EGI-certified SE implementations (Disk Pool Manager, dCache and StoRM) support SRM which also ensures compatibility with various client tools. Currently, not all resource centers supporting medical image processing VOs got certified for operation in the EGI and hence information about storage resources is not published in the global resource indexes.

Dedicated information index was set up within the medgrid $\mathrm{VO}$ which collects information from all relevant SEs as well as LFC services. This allowed implementing web services for storage interaction relying on standard EMI client tools and libraries for data management.

Access to the SEs is controlled on the VO membership basis. Permissions to access certain files are deduced from VOMS [7] groups and roles that get assigned to users and services by the VO managers. A highly available VOMS service ensures data access permissions integrity because all the Storage Elements are configured to download corresponding information from VOMS.

To facilitate data exchange between medical information systems and portals and the grid storage infrastructure, web services were developed and deployed in the UNG.

The file exchange web-service (GW-FEX) [12] in Fig. 1 provides a gateway for exchanging data between web and grid infrastructure. The service is implemented as asynchronous request processor and provides RESTful interface for submitting new data transfer requests and querying status of previously submitted ones. Representational State Transfer (REST) [13] is a modern architectural concept of interactive multimedia (hypermedia) distributed systems.

From the web-site, the service is able to download and upload files to FTP and WebDAV (Web Distributed Authoring and Versioning) [14] shares. In the common usage scenario, web-portal has a spool folder shared by one of the supported 
protocols accessible from the gateway service host. Requests are initiated by the web-portal. After successful completion of put-to-grid request, the gateway service returns grid identifier of the stored file which can be used later to submit pull-from-grid request for getting data back to the web-portal. Requests can be grouped to be processed in a batch. From the grid-side, the service operates with its own service certificate which is used to get authorization from VOMS and to access LFC data catalogs and SRM Storage Elements.

Permissions for a particular gateway are controlled by VOMS attributes assigned to the service. Standard EMI data management tools are used to implement data transfers and metadata registration in the grid infrastructure.

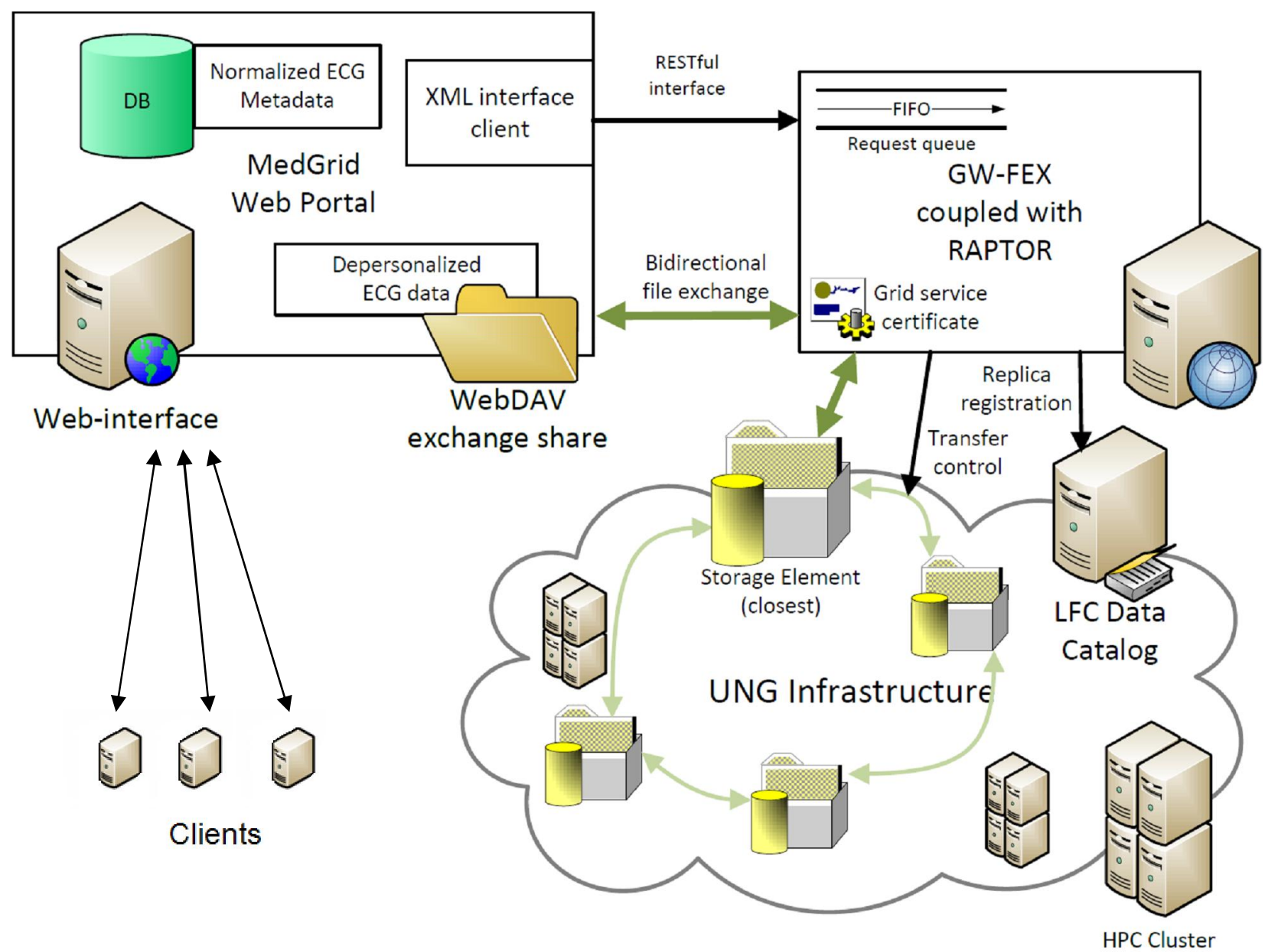

Fig. 1 - Web-service diagram.

Automatic data replication for maintaining high availability of data in the grid infrastructure is achieved by complementing the GW-FEX service with Robot for Autonomous Precisely Tunable Operation of Replication (RAPTOR) service [15]. RAPTOR service shares the request queue with the gateway service and continuously crawls through LFC catalog to verify replication policies for data stored in the grid. If some file was declared to be highly available with minimal number of replicas defined in the policy, then RAPTOR verifies availability of existing replicas and submits requests to produce new replicas when needed. This ensures that some particular file in the catalog minimally has a particular number of online replicas.
Consolidated deployment of GW-FEX and RAPTOR services provides a simple web service interface to reliable storage infrastructure for webportals and hiding all the complexity of grid infrastructure internal operations. Consolidated installation of both services was employed by medgrid VO web-portal [7] to facilitate storage of ECG data.

The whole picture of interaction between web services and grid services is shown in Fig. 1. File exchange service places new data received from web portal on the closest grid storage element which is located on the same site. After successful upload, an entry for a file is created in the LFC data catalog and grid unique ID (GUID) gets assigned to the file. 
From now, the file can be identified and accessed from the grid services.

Data catalog record eventually gets checked for conformance to a replication policy by the replication service. If having a single replica is not enough, requests for creating copies of the file on different SEs are pushed to the request queue. After successful replication, an entry in the LFC catalog is updated and new data locations are populated in the file record.

The RAPTOR service not just verifies a replicas but also checks its health. In the case of faulty SE data gets one more replica on a healthy one.

\section{AMGA SERVICE}

The ARDA Metadata Grid Application project (AMGA) [16] has emerged as a general purpose metadata catalogue service which allows attaching any relationally organized information to files stored in the grid infrastructure. AMGA can also be used as independent grid-enabled RDBMS service.

For medicine grid-enabled applications, AMGA is used for storing metadata found in DICOM images as well as electrocardiograms and other specific formats. Ability to select a dataset based on some properties like patient age, living location etc. is critical for population studies.

Besides providing a low-level API for database access, AMGA features a set of components for building web-based interfaces to metadata databases. This functionality had used in several projects across UNG that rely on central AMGA catalogue instance coupled with central grid file catalogue.

\section{CLIENTS}

Clients are specially designed to be compatible with different operating systems, to be independent of grid middleware and to be easily extensible. Portability is achieved by employment of portable software and libraries: GUI was implemented in Microsoft .NET; network components are implemented using open source software, like cURL library [17] and GNU compiles; database access is implemented with ODBC interface; DICOM parsing is provided in the portable DICOM Toolkit library [18].

User client in Fig. 2 consists of several modules interacting with each other and implemented as different processes or threads. Number of modules may be different depending task to solve. For medical application the main module is graphical user interface (GUI). It performs visualization of images in DICOM format. Visualizing module supports images with more then 8-bits pixel depth, manual and automatic changing of brightness scaling window position and width, multi-frame mode with animations. GUI also provides means to work with images data, open and save examinations, lookup in local database, implements functions associated with data transfer to/from grid infrastructure. New functions may be added by external software modules. Data exchange with grid infrastructure is performed by network agent. Some functions of DICOM data parsing are performed by DICOM utility. Data anonymizing/deanonymizing is performed by anonymizer utility.

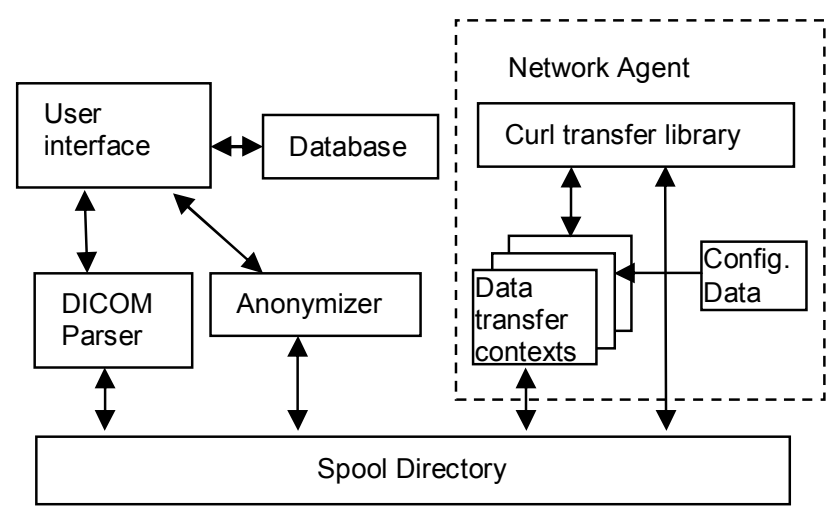

Fig. 2 - Client components diagram.

Interaction between modules is performed using spool directories on file-system and local database. Interactions via spool directories are used for transactions that have no references to other transactions or operate with data that should not be saved permanently. Data consistency in spool directories is provided by using simple requestresponse locking technique. Consumer creates temporary request file and adds information to it. When finished temporary request file is copied to main request file and after that temporary file is deleted. Provider periodically or by notification checks spool directory for main request files that have no temporary files. While request is executed its request file is used as $\log$ and transaction database. Information is added into request file when necessary. When execution is complete its request file is copied to response file and request file is deleted. Consumer checks spool directory for response files without request files, acquires request processing results and deletes the response file. Such scheme being correctly used provides data consistency even in the cases of software or hardware failures, network transfer aborts etc. This exchange method does not require any additional libraries and is very portable.

Interactions via database are used for transactions that may be simultaneously accessed by different modules, when some records should be saved permanently or when search capabilities are necessary. 
Anonymizer is an external software module that provides removal of patient's personal data from DICOM images before transferring data to the grid for storage and restoration of personal data after transferring images from the grid. Anonynizer is called by GUI for all images to be transferred.

For each image transferred to the grid anonymizer inserts patient ID into local database if it does not exist there and reads patient alias from database. This alias is written into anonymized DICOM file. Then values of DICOM tags needed to be anonymized are cleared from anonymized file and whole DICOM header of original file gets saved in the local database. Request to send anonymized file is passed to network agent. After transfer to the grid network agent returns response file to user interface which deletes all temporary data files and inserts grid file location URL into the local database. When images are transferred back from the grid storage, anonymizer restores the whole DICOM header from the database using patient alias. Image files that have beem transferred to the grid may be deleted on the client and restored later from the grid. Tags that should be anonymized are configured via client GUI and may be changed.

This approach provides complete anonymizing of data on client side and thus eliminates transfer of personal data. Personal information may be restored only on the client that performed anonymizing. Shortcoming of this approach is treatment of the same patient on different clients as different persons. This problem may be solved by exchanging of local database content between authorized clients.

Network agent provides interaction with grid and web services like data transfers, job submissions, search queries etc. It is implemented as multiprotocol daemon process that interacts with GUI and other client components via spool directory. Network agent is object oriented so new protocols may be easily added by redefinition of some callback functions in job context classes.

Network agent runs continuous loop where all job contexts' instances are executed. Now two job contexts concerned with data exchange with the grid infrastructures are implemented. Multiple instances of each job context are allowed to run in parallel so many data transfers to and from the grid may be performed simultaneously. Network transfer management is performed by cURL library. Each job context passes authorization to web service using login/password or personal user certificate. Then data is transferred via HTTP POST request. After transfer is complete, the response a file is passed to the GUI. This file contains status information, transfer statistics and grid URL of file for send request.

\section{MEDICAL APPLICATIONS}

The proposed clients are used to implement tools (Fig. 3) for archiving of medical images from different Healthcare institutions in Ukraine. The main goal of the project is creation of large archive of anonymized DICOM images of different modalities. One of such archives is created today by The Institute for Scintillation Materials National Academy of Sciences of Ukraine to store scintigraphic and SPECT images from gammacameras in different nuclear medicine institutions of Ukraine.

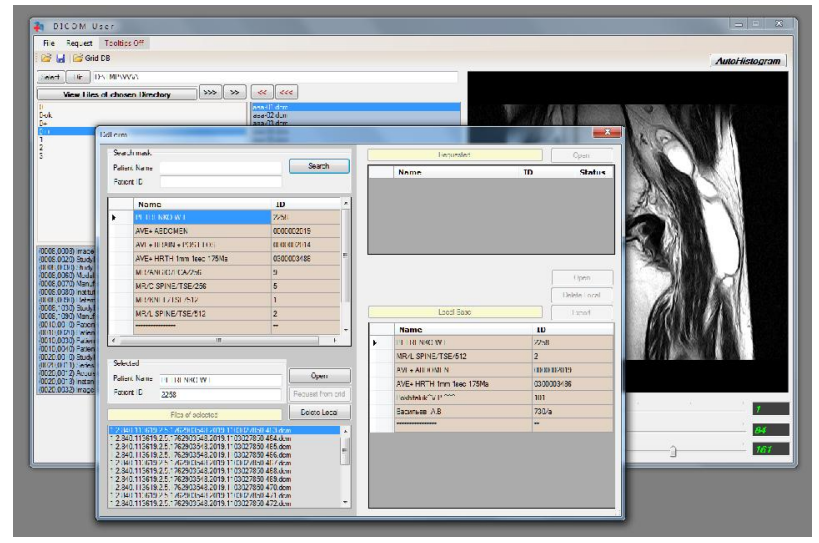

Fig. 3 - Medical application GUI screenshot

Direct usage of MDM or other grid DICOM tools for such tasks requires installation of grid middleware on workstations or access to PACS (Picture Archiving and Communication System) DICOM servers from grid sites. The first task is complicated and the second task is not usually possible because LANs of medical institutions are usually separated from the Internet. To simplify the task of the grid archive access, DICOM images are transferred to the grid infrastructure from physicians' workstations that can access local DICOM images and Internet access via firewall or proxy server by means of proposed clients.

Now there are three large projects in UNG devoted to medical images archiving. The first one is creation of emission tomography images archive in the Institute for Scintillation Materials National Academy of Sciences of Ukraine. This archive runs web-service in the Institute for Cybernetics National Academy of Sciences of Ukraine and has replicated storages in UNG. Kiev Municipal Heart Centre and Amosov National Institute of Cardio-pulmonary Surgery use this archive for reliable medical images storing.

Another project that use the services developed is creation of electrocardiograms (ECG) archive in the Institute for Problems of Mathematical Machines and systems National Academy of Sciences of 
Ukraine. This archive is used in several medical institutions of Ukraine supporting medgrid $\mathrm{VO}$ [8].

Currently there are about 20 thousands of ECGs accumulated in the archive. Single ECG file has nearly a few megabytes in size and hence there was implemented aggressive data replication policy that specify creation of replicas of all ECGs on all accessible grid-storages [12].

Such approach enables for meeting all needed requirements for carrying out population-based studies on top of geographically distributed gridsites [19].

The third project is creation of electroencephalograms (EEG) archive in UNG by the Bogomolets Institute of Physiology National Academy of Sciences of Ukraine. This archive is used in National Taras Shevchenko University of Kyiv, Ukraine and in The University of Vermont, USA for archiving human and rat EEG data for scientific research. This project also has the aim to create multimodal database and knowledge dataset of physiological data.

Another intended application of this client software is employment of grid computing resources for computation-intensive image reconstruction [20], fusion, recognition etc. There plans to create differential diagnostic service based on SPECT images, and USI images [21], procession of ECG and EEG data etc.

Now described clients lack capabilities for searching data in the grid. This function is planned to be implemented by interfacing AMGA Metadata Catalog grid services and integration with MDM in more distant future.

\section{APPLICATIONS FOR MASSIVE SIMULATIONS}

Proposed storage services also used for creation of massive scientific simulations results databanks. The first databank was created in the National Scientific Center for Medical and Biotechnical Research National Academy of Science of Ukraine $[4,5]$. This data archive contains results of nonlinear dynamics simulations for different systems including neurons systems.

This archive contains about 400000 dynamics trajectories for $1 \mathrm{~d}, 2 \mathrm{~d}$ and $3 \mathrm{~d}$ systems (Fig. 4a) simulated in UNG. Aggregation diagrams Fig. 4b are built from data in these archives. Each pixel of such diagrams corresponds to one trajectory in different parameters space. Clicking in appropriate place at the aggregation diagram gives a page with extended parameters of trajectory. Besides image representation diagram also is represented as table with sorting capabilities. Searching is possible on web page using web browser search functions.

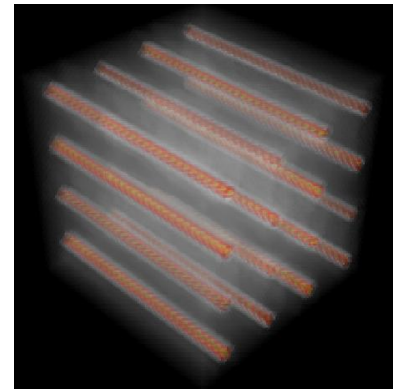

a)

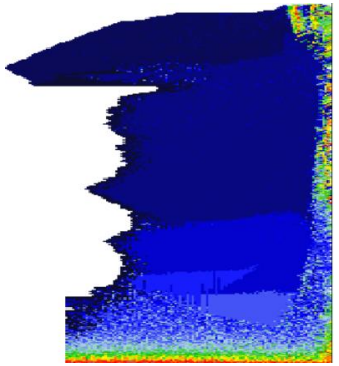

b)
Fig. 4 - Archive of non-linear dynamics data: result of 3d system simulation (a), aggregation diagram (b).

One of the most successful UNG applications in the VO MolDynGrid [3] uses the replication services developed for storing simulation results and initial parameters of molecular dynamics in grid infrastructure for further complex analysis.

Currently there are about one thousand trajectories of the molecular dynamics. Because of huge size of one trajectory (usually from 10 GB to $100 \mathrm{~GB}$ for typical biological objects studies in virtual laboratory), a decision was taken to have from 2 to 3 online replicas of it on the grid storages available for VO.

Using balanced policy in the replication scheme allowed providing enough level of data redundancy, reducing load on grid storage elements and network communications.

\section{CONCLUSION}

Numerous successful applications of proposed grid-services and clients for archiving of medical images, EEG and ECG data, dynamical simulation results prove that presented tools provide means for quick creation and usage of grid archives. Tools operate directly from users workstations and clusters possibly eliminating complicated procedures of grid middleware installation, certificate obtaining etc. Built-in support for high performance data transfers, data anonymization and extensibility are distinctive features of this software suite. Future plans include more tight integration of proposed storage services with computing and data search facilities and creation of grid expert systems on using proposed storage services.

\section{ACKNOWLEDGMENTS}

Funding for the project was provided within The Ukrainian State Scientific and Technical Programme on Development and Application of Grid Technologies in 2009-2013 by State Agency on Science, Innovations and Informatization of Ukraine and National Academy of Sciences of Ukraine. All 
research and development was held in Ukrainian National Grid (UNG) infrastructure on clusters of Information and Computer Centre National Taras Shevchenko University of Kyiv and National Scientific Centre for Medical and Biotechnical Research National Academy of Sciences, Ukraine.

\section{REFERENCES}

[1] I. Foster and C.Kesselman, The Grid: Blueprint for a New Computing Infrastructure, Morgan Kaufmann, 1999.

[2] M. Zynovyev, S. Svistunov, Y. Boyko, and O. Sudakov, Ukrainian grid infrastructure: Practical experience, Proceedings of the $4^{\text {th }}$ IEEE Workshop on Intelligent Data Acquisition and Advanced Computing Systems: Technology and Applications IDAACS'2007, September 6-8, 2007, Dortmund, Germany, pp. 165-169.

[3] A. O. Salnikov, I. A. Sliusar, O. O. Sudakov, O. V. Savytskyi, A. I. Kornelyuk, MolDynGrid virtual laboratory as a part of Ukrainian Academic Grid infrastructure, Proceedings of the $5^{\text {th }}$ IEEE International Workshop on Intelligent Data Acquisition and Advanced Computing Systems: Technology and Applications IDAACS'2009, Rende (Cosenza), Italy, September 21-23, 2009, pp. 237-240.

[4] A. Salnikov, R. Levchenko, O. Sudakov, Integrated grid environment for massive distributed computing in neuroscience, Proceedings of the $6^{\text {th }}$ IEEE International Conference on Intelligent Data Acquisition and Advanced Computing Systems: Technology and Applications IDAACS'2011, Prague, Czech Republic, September 15-17, 2011, pp. 198-202.

[5] O. E. Omel'chenko, M. Wolfrum, S. Yanchuk, Y. L. Maistrenko, O. Sudakov, Stationary patterns of coherence and incoherence in twodimensional arrays of non-locally-coupled phase oscillators, Physical Review E, Vol. 85, Issue 3, 2012, Paper ID 036210.

[6] O. Sudakov, M. Kononov, Ie. Sliusar, A. Salnikov, User clients for working with medical images in Ukrainian grid infrastructure, Proceedings of the $7^{\text {th }}$ International IEEE Conference on Intelligent Data Acquisition and Advanced Computing Systems: Technology and Applications IDAACS'2013, Berlin September 12-14, 2013, pp. 705-710.

[7] Virtual organization membership service of Ukrainian national grid (UNG), National Taras Shevchenko University of Kyiv, 2011. [Online]. Available: http://grid.org.ua/voms/

[8] Medical Grid-system for population research in the field of cardiology with electrocardiogram database 2012. [Online]. Available http://medgrid.immsp.kiev.ua/

[9] Peter Mildenberger, Marco Eichelberg, and Eric Martin, Introduction to the DICOM standard, European Radiology, Vol. 12, Issue 4, 2002, pp. 920-927.

[10] Johan Montagnat, Ákos Frohner, Daniel Jouvenot, Christophe Pera, Peter Kunszt, Birger Koblitz, Nuno Santos, Charles Loomis, Romain Texier, Diane Lingrand, Patrick Guio, Ricardo Brito Da Rocha, Antonio Sobreira de Almeida, Zoltán Farkas, A secure grid medical data manager interfaced to the gLite middleware, Journal of Grid Computing (JGC), Vol. 6, Issue 1, Kluwer, March 2008, pp. 4559, [Online]. Available http://modalis.i3s.unice.fr/softwares $/ \mathrm{mdm} /$

[11] A. Shoshani, A. Sim, J. Gu, Storage resource managers: essential components for the grid, Grid Resource Management: State of the Art and Future Trends. Ed. by Jan Weglarz, Jarek Nabrzyski, Jennifer M. Schopf, Boston: Kluwer Academic Publishers, 2003, Vol. 64 of International Series in Operations Research \& Management Science, pp. 329-348, ISBN: 978-1402075759.

[12] I. Sliusar, M. Volzheva, Using grid infrastructure as a distributed fault-tolerant data storage for web services, Proceedings of the Second International Conference on Cluster Computing CC'2013, Ukraine, Lviv, June 3-5, 2013, pp. 266-268.

[13] R. T. Fielding, R. N. Taylor, Principled design of the modern Web architecture, $A C M$ Transactions on Internet Technology, Vol. 2, Issue 2, May 2002, pp. 115-150.

[14] L. Dusseault, HTTP extensions for Web distributed authoring and versioning (WebDAV), Request for Comments: 4918. [Online]. Available http://tools.ietf.org/html/rfc4918

[15] I. A. Sliusar, Automatic replication service for maintaining high availability of data in the grid-infrastructure, Control Systems and Machines, No. 4, 2012, pp. 63-69. (in Ukrainian)

[16] B. Koblitz, N. Santos, V. Pose, The AMGA metadata service, Journal of Grid Computing, Springer Netherlands, Vol. 6, No. 1, 2008, pp. 61-76.

[17] Libcurl: the multiprotocol file transfer library, 2013 [Online]. Available http://curl.haxx.se/

[18] DCMTK: DICOM Toolkit, 2013 [Online]. Available http://dicom.offis.de/

[19] T. Romanenko, V. Vishnevskiy, A. Boretskiy, Features of launching grid task with low computing time and big data transfers in a 
distributed repositories of "Medgrid" VO, Proceedings of the International Conference on Parallel and Distributed Computing Systems PDCS'2013, Ukraine, Kharkiv, March 13-14, 2013, pp. 266-268.

[20] M. V. Kononov, O. A. Nagulyak, A. V. Netreba and A. A. Sudakov, Reconstruction in NMR by the method of signal matrix pseudoinversion, Radioelectronics and Communications Systems, Vol. 51, Num. 10, October 2008, pp. 531-533.

[21] M. K. Novoselets, S. P. Radchenko, V. A. Tsubin, O. M. Gridko, Ultrasound introscopic image quantitative characteristics for medical diagnostics and refinements of physical noise rise reasons, Proceedings of the Medical Imaging, 1994, SPIE, Physics of Medical Imaging, Vol. 2163, 1994, pp. 442446.

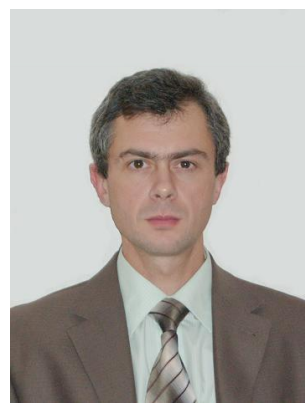

Oleksandr O. Sudakov Candidate of physical and mathematical sciencies (Ph.D.). Associate Professor of Medical Radiophysics Department of Radiophysics Faculty, Head of Parallel Computing Laboratory at Information and Computer Center Taras Shevchenko National University of Kyiv. Graduated from Radiophysics Faculty Taras Shevchenko Kyiv University in 1996. Ph.D. thesis in Procession of magnetic resonance tomography signals in 2002. Scientific interests: high performance computing, physical processes in biological systems.

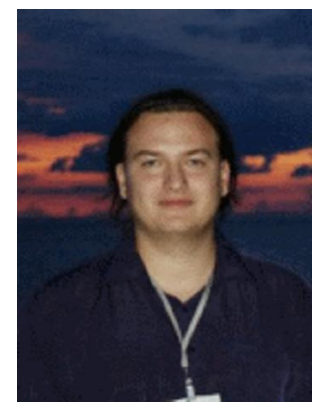

Andrii O. Salnikov, Assistant lecturer at Computer Engineering Department of Radiophysics Faculty, Senior engineer at Parallel Computing Laboratory of Information and Computer Center of Taras Shevchenko National University of Kyiv. Post-graduate of techical sciences.

Scientific interests: grid and high performance computing.

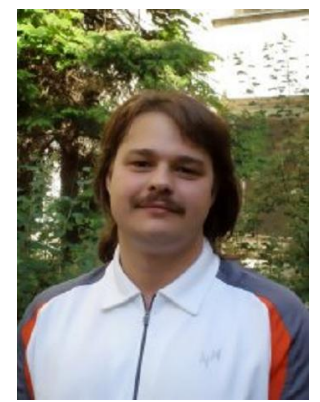

levgen A. Sliusar, Assistant lecturer at Computer Engineering Department of Radiophysics Faculty, Senior engineer at Parallel Computing Laboratory of Information and Computer Center of Taras Shevchenko National University of Kyiv. Post-graduate of technical sciences.

Scientific interests: grid and high performance computing.

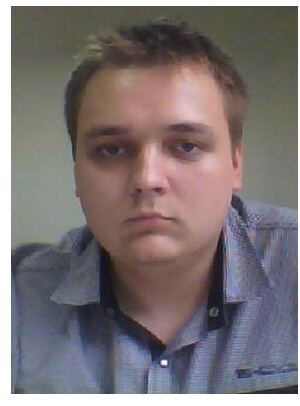

Oleksandr F. Boretskyi, Master of physical and mathematical sciences, Postgraduate student of technical sciences, Engineer at Parallel Computing Laboratory of Information and Computer Center of Taras Shevchenko National University of Kyiv. Scientific interests: grid and high performance computing. 